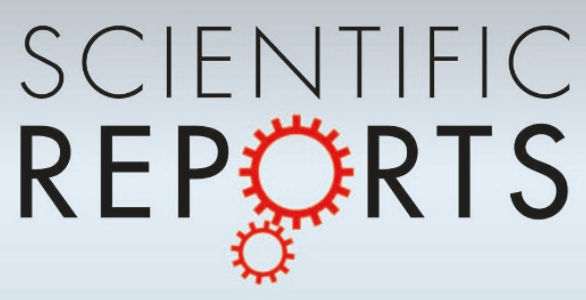

OPEN

SUBJECT AREAS:

DIAGNOSTIC MARKERS

IMMUNOLOGICAL TECHNIQUES

Received

17 January 2014

Accepted

3 March 2014

Published

18 March 2014

Correspondence and requests for materials should be addressed to S.K.V. (sandeep. kumar.vashist@hsgimit.de)

\title{
One-step antibody immobilization-based
} rapid and highly-sensitive sandwich ELISA procedure for potential in vitro diagnostics

\author{
Sandeep Kumar Vashist ${ }^{1,2}$, E. Marion Schneider ${ }^{3}$, Edmond Lam ${ }^{4}$, Sabahudin Hrapovic ${ }^{4}$ \& John H. T. Luong ${ }^{5}$
}

${ }^{1}$ HSG-IMIT - Institut für Mikro- und Informationstechnik, Georges-Koehler-Allee 103, 79110 Freiburg, Germany, ${ }^{2}$ Laboratory for MEMS Applications, Department of Microsystems Engineering -IMTEK, University of Freiburg, Georges-Koehler-Allee 103, 79110 Freiburg, Germany, ${ }^{3}$ Sektion Experimentelle Anaesthesiologie, University Hospital Ulm, Albert Einstein Allee 23; 89081 Ulm, Germany, ${ }^{4}$ National Research Council Canada, Montreal, Quebec, Canada H4P 2R2, ${ }^{5}$ Innovative Chromatography Group, Irish Separation Science Cluster (ISSC), Department of Chemistry and Analytical, Biological Chemistry Research Facility (ABCRF), University College Cork, Cork, Ireland.

An improved enzyme-linked immunosorbent (ELISA) assay using one-step antibody immobilization has been developed for the detection of human fetuin A (HFA), a specific biomarker for atherosclerosis and hepatocellular carcinoma. The anti-HFA formed a stable complex with 3-aminopropyltriethoxysilane (APTES) by ionic and hydrophobic interactions. The complex adsorbed on microtiter plates exhibited a detection range of $4.9 \mathrm{pg} \mathrm{mL}^{-1}$ to $20 \mathrm{ng} \mathrm{mL}^{-1} \mathrm{HFA}$, with a limit of detection of $7 \mathrm{pg} \mathrm{mL}^{-1}$. Furthermore, an analytical sensitivity of $10 \mathrm{pg} \mathrm{mL}^{-1}$ was achieved, representing a 51-fold increase in sensitivity over the commercial sandwich ELISA kit. The results obtained for HFA spiked in diluted human whole blood and plasma showed the same precision as the commercial kit. When stored at $4^{\circ} \mathrm{C}$ in $0.1 \mathrm{M}$ phosphate-buffered saline (PBS, pH 7.4), the anti-HFA bound microtiter plates displayed no significant decrease in their functional activity after two months. The new ELISA procedure was extended for the detection of C-reactive protein, human albumin and human lipocalin-2 with excellent analytical performance.

\footnotetext{
LISA is the gold standard of in vitro diagnostics (IVD) during the last five decades for analysis of biomarkers and important analytes in healthcare and diversified analytical settings. With over 300,000 peer-reviewed articles to date, ELISA-based technologies have open up a lucrative, commercial market. Despite ongoing developments in immunosensors, labs-on-chips, and microfluidic and point-of-care technologies, ELISA with high throughput and omnipotent nature has been unmatched in reliability for the monitoring and management of disease markers. It is still the most widely used immunoassay format by pharmaceutical industries for routine monitoring of drugs and drug impurities (e.g. Chinese hamster ovary protein and monocyte chemotactic protein). Competing immunoassay technology must be compared to ELISA for precision and other analytical parameters.

Defined plasma biomarkers are of unique diagnostic relevance for early preventive intervention in chronic inflammatory diseases, highly prevalent in the Western world. One of those biomarkers is HFA where a highly sensitive and rapid assay is of value when combined with sensitive measurements of C-reactive protein ${ }^{1}$. HFA is a product of the liver and its concentration decreases during the acute phase reaction. Due to its anti-inflammatory properties by counteracting proinflammatory cytokine production, quantification in body fluids is highly relevant in guiding diagnostics and therapy of infection-independent diseases of liver, heart and vasculature. In vivo, HFA functions as an inhibitor of soft tissue calcification and is a specific biomarker for hepatocellular carcinoma ${ }^{2}$ and atherosclerosis ${ }^{3}$, and associated with arthritis $^{4}$, cardiovascular diseases ${ }^{5-7}$, malaria $^{8}$, diabetes ${ }^{9}$, and metabolic syndrome $^{10}$, as well as neurological diseases such as multiple sclerosis ${ }^{11}$.

The last two decades have witnessed considerable advances in the development of improved immunoassay procedures for IVD including improved antibody (Ab) immobilization chemistries, signal enhancement strategies using micro-/nanomaterials or polymers, novel lab-on-a-chip technologies, biosensors and novel immunoassay formats. In all cases, the development of an appropriate Ab immobilization strategy is a critical requirement
} 
that significantly affects the analytical performance of an immunoassay ${ }^{12-14}$. Examples range from adsorption, oriented binding using fragment crystallizable $\left(\mathrm{F}_{\mathrm{c}}\right)$ proteins, covalent binding using crosslinkers, site-directed immobilization, and non-covalent binding ${ }^{15-21}$. However, most of these procedures employ a complex multi-step procedure involving costly crosslinking agents.

This article describes a simple immobilization-based sandwich ELISA procedure for the development of rapid, low-cost and highly-sensitive IVD kits. The new immobilization format (NIF) only involves the dilution of the antibody in APTES to form a stable complex. APTES-polymer/Ab complexes sorbed on the microtiter plate (MTP) will be evaluated with respect to detection limit, analytical sensitivity, storage stability and its applicability for detecting HFA in human whole blood and plasma.

\section{Results}

In this procedure, the capture Ab was admixed with $1 \%(\mathrm{v} / \mathrm{v})$ APTES $(1: 1 \mathrm{v} / \mathrm{v}$ mixture), dispensed into the MTP wells and incubated for $30 \mathrm{~min}$ (Figure 1). Under the optimized condition (Supplementary Figures S1a-b), HFA from 4.9 to $20,000 \mathrm{pg} \mathrm{mL}^{-1}$ was detected with linearity of 156 to $20,000 \mathrm{pg} \mathrm{mL}^{-1}$ (Figure 2a). The estimated limit of detection (LOD) and analytical sensitivity were $7 \mathrm{pg} \mathrm{mL}^{-1}$ and $10 \mathrm{pg}$ $\mathrm{mL}^{-1}$, respectively. The intraday variability of five assay repeats (in triplicate) in a single day ranged from 1.2 to 8.5 , while the interday variability of five assay repeats (in triplicate) on five consecutive days was between 2.1 to 10.2. With a maximal half-effective concentration $\left(\mathrm{EC}_{50}\right)$ of $2.6 \mathrm{ng} \mathrm{mL} \mathrm{m}^{-1}$, the NIF was highly specific to HFA without any interference from immunological reagents in different process controls (Figure 2b).

The NIF was compared with a commercial ELISA kit and a typical sandwich ELISA procedure with covalently cross-linked $\mathrm{Ab}$ on APTES-functionalized surfaces ${ }^{17,22}$. All immunoassays were performed under the same conditions with the same assay components to minimize experimental variability. The NIF outperformed conventional immobilization format (CIF)-based sandwich ELISA with 28fold faster $\mathrm{Ab}$ immobilization and 51-fold more sensitivity. Compared to the covalent immobilization format (CovIF) that involves the covalent crosslinking of Ab to the APTES-functionalized MTP, it is still 5fold faster in terms of $\mathrm{Ab}$ immobilization and 3-fold more sensitive (Table 1, Supplementary Table S1). The Ab immobilization density of the NIF estimated by bicinchoninic acid protein assay was compared favorably with the results obtained by the CIF, CovIF and a new covalent immobilization format (NCIF) (which involves covalent antibody immobilization involving 1-ethyl-3-(3-dimethylaminopropyl) carbodiimide hydrochloride (EDC)-activated anti-HFA Ab diluted in APTES (for covalent binding of Ab to APTES into KOH-pretreated MTP wells) (Supplementary Figure S2). Hence, the NIF is the most sensitive with minimal reagents and analysis time for the detection of HFA compared to various ELISA formats.

Central to the NIF is the sorption of the APTES-polymer/Ab complex to the MTP surface. To elucidate the binding of the complex to a polystyrene (PS) MTP in the absence of a crosslinker, immunoglobulin $\mathrm{G}$ (IgG) bovine was used as the model. PS beads were immersed in a solution of APTES/IgG in PBS buffer $(0.2 \mathrm{mg} / \mathrm{mL}$ IgG and $0.5 \%$ APTES) for $30 \mathrm{~min}$. The IgG/APTES solution was decanted and the resulting PS beads were washed five times with PBS buffer, followed by three times with deionized water to remove residual $\mathrm{Na}_{2} \mathrm{HPO}_{4}$ and $\mathrm{KH}_{2} \mathrm{PO}_{4}$ salts that will interfere with Fourier transform infrared spectroscopy (FTIR) and energy-dispersive X-ray (EDX) analyses. The IgG concentration was increased to provide greater peak intensity for IgG bands in the FTIR spectra for facilitating bonding elucidation. In aqueous solution, APTES reacts with the free hydroxyls of an oxidized substrate by $\mathrm{S}_{\mathrm{N}} 2$ exchange with loss of ethanol. The next step is condensation that leads to polymerization when an APTES molecule forms a siloxane with its neighboring APTES (Figure 1).

Figure 3 shows five FTIR spectra: PS, PS+APTES, PS+APTES+ IgG, PS + IgG and IgG. PS exhibits the usual FTIR peaks consistent for all PS derivatives. Upon addition of APTES, the spectrum shows the Si-O-Si character at $1154 \mathrm{~cm}^{-1}$, with $1671 \mathrm{~cm}^{-1}$ and a broad $3400 \mathrm{~cm}^{-1}$ peaks for the primary $\mathrm{NH}$, confirming the presence of APTES and its polymerization ${ }^{17,23}$. For derivatives with IgG, additional bands for the amides at 1656, $1548 \mathrm{~cm}^{-1}$ with broad $\mathrm{OH}, \mathrm{NH}$
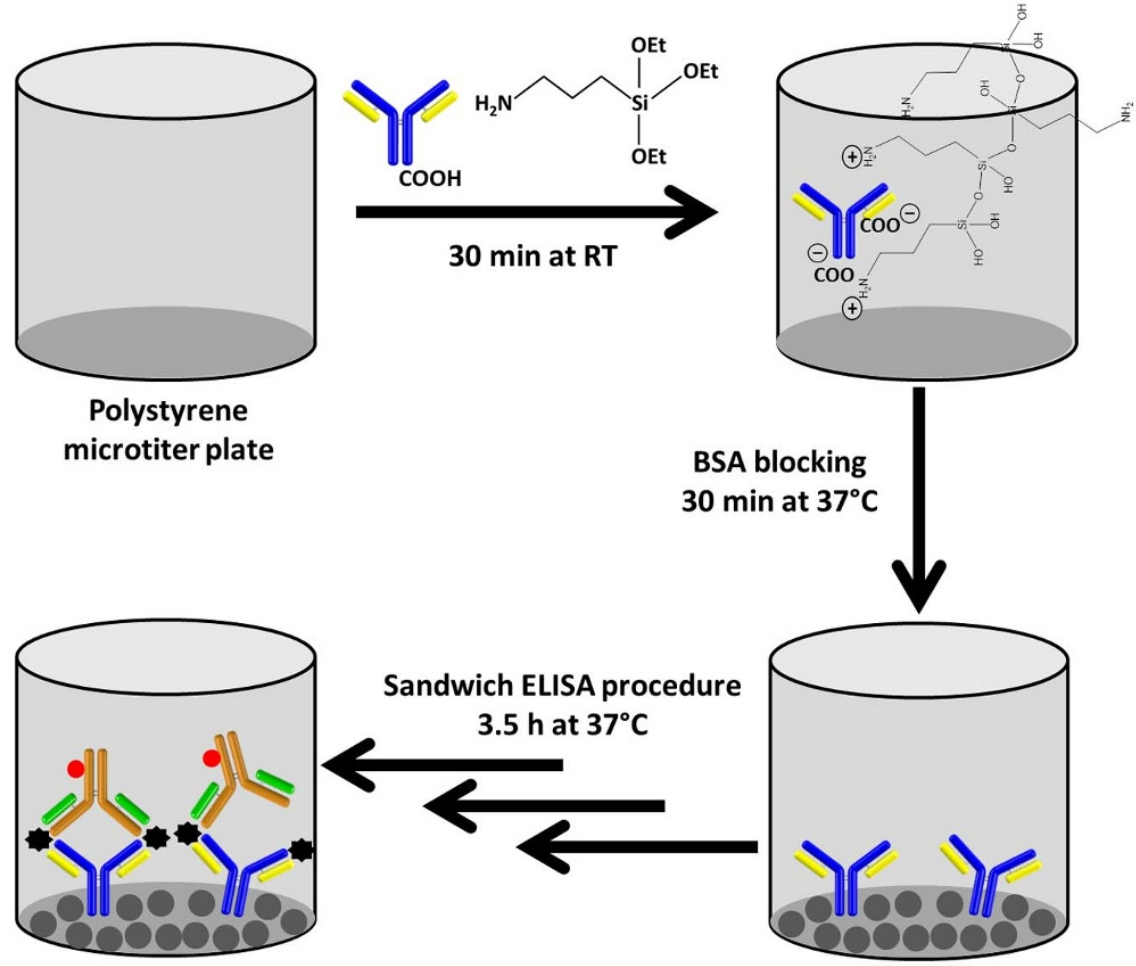

Figure 1 One-step antibody immobilization-based sandwich ELISA procedure for the detection of human fetuin A (HFA). 
(a)

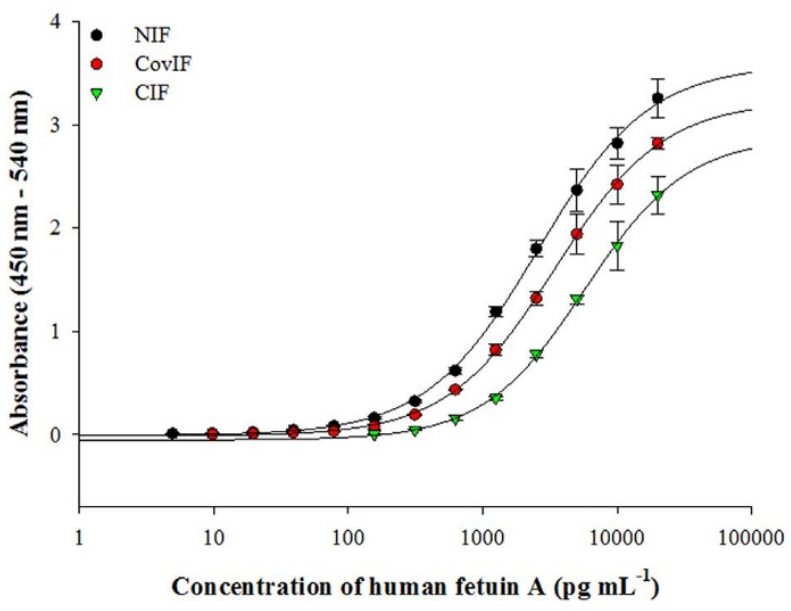

(c)

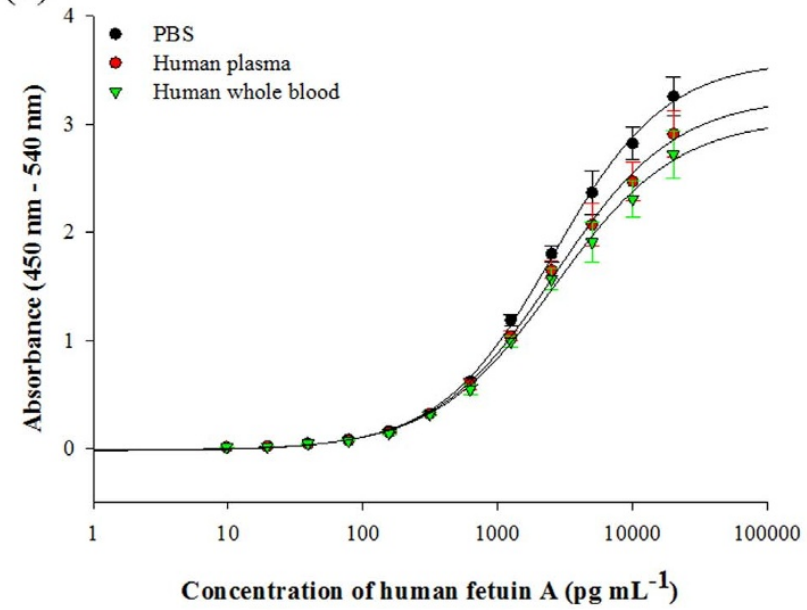

(b)

(d)
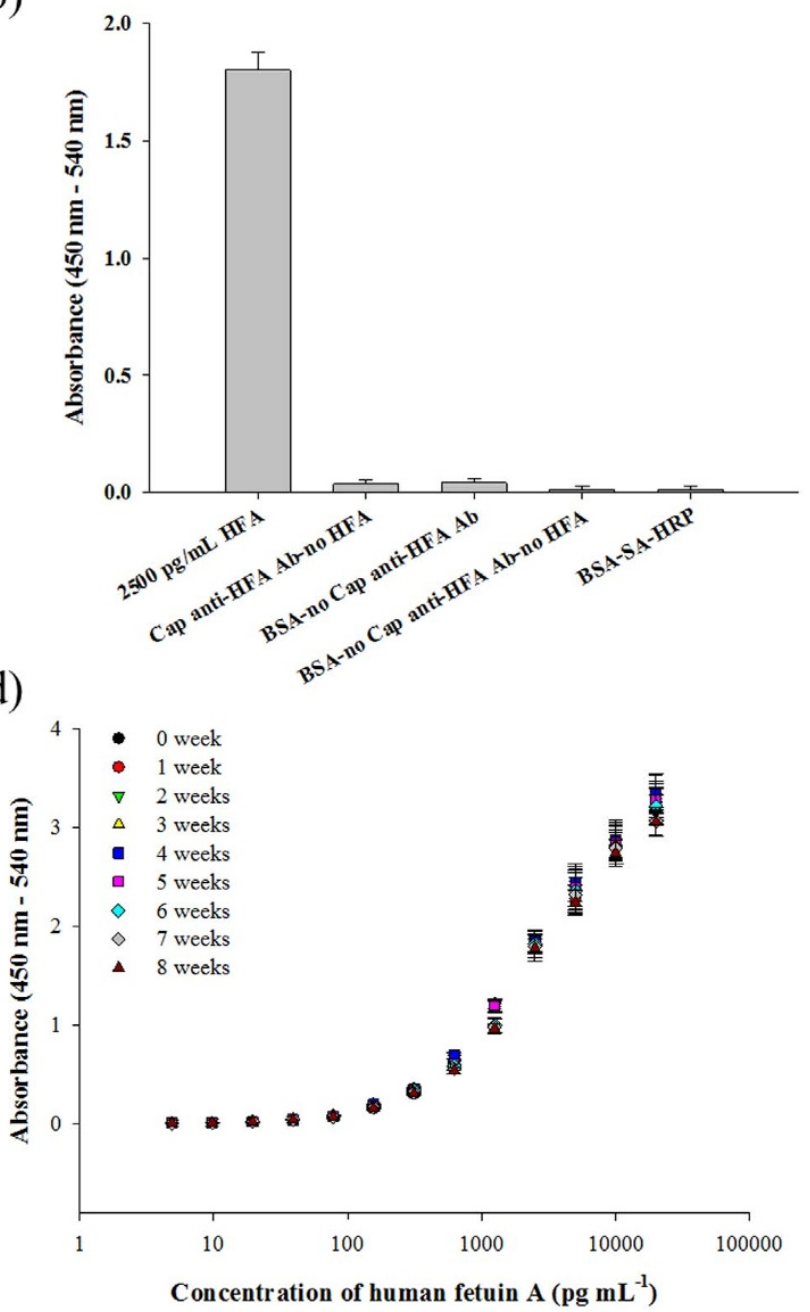

Figure $2 \mid$ One-step antibody (Ab) immobilization-based sandwich ELISA. (a) Detection of HFA by the NIF, covalent immobilization format (CovIF)based $^{5,9}$ and conventional immobilization format (CIF)-based (passive adsorption-based) sandwich ELISA procedures. (b) Specific HFA detection with respect to various experimental process controls. (c) Detection of HFA spiked in PBS (0.1 M, pH 7.4), diluted human whole blood and diluted human plasma. (d) Detection of HFA by the anti-HFA Ab-bound MTPs stored in $0.1 \mathrm{M} \mathrm{PBS}, \mathrm{pH} 7.4$ at $4{ }^{\circ} \mathrm{C}$ for 8 weeks. All experiments were done in triplicate with the error bars representing the standard deviation.

bands centered at $3305 \mathrm{~cm}^{-1}$ that match with the IgG spectrum, although the two peaks for IgG at 1387 and $1231 \mathrm{~cm}^{-1}$ have diminished in those PS derivatives. The bands associated with the $\mathrm{PO}_{4}$ buffer salts are not present in the spectra to obscure the identity of the bands of interest. EDX analysis of the materials shows that the $\mathrm{Si}$ wt.\% content is $0.07 \%$ for the PS derivatives with APTES with the absence of $\mathrm{Na}, \mathrm{K}$ and $\mathrm{P}$ from the PBS buffer salts, confirming the validity of the water-wash procedure prior to FTIR acquisition.

Table 1 Analytical comparison of the NIF with the conventional immobilization format (CIF) and covalent immobilization format (CovIF)based $^{5,9}$ sandwich ELISAs for the detection of HFA

\begin{tabular}{|c|c|c|c|}
\hline & $\mathrm{NIF}^{1}$ & CovlF $^{2}$ & $\mathrm{ClF}^{3}$ \\
\hline Assay duration $(\mathrm{h})$ & $\sim 4$ & $\sim 6$ & $\sim 20$ \\
\hline LOD $\left(p g \mathrm{~mL}^{-1}\right)$ & 7 & 12 & 226 \\
\hline Analytical sensitivity (pg mL ${ }^{-1}$ ) & 10 & 30 & 510 \\
\hline $\begin{array}{l}\mathrm{EC}_{50}\left(\mathrm{ng} \mathrm{mL}^{-1}\right) \\
\% \mathrm{CV}\end{array}$ & 2.6 & 3.4 & 5.8 \\
\hline Assays on various substrates & Yes & Yes & No \\
\hline Requirement for crosslinkers & No & Yes & No \\
\hline
\end{tabular}




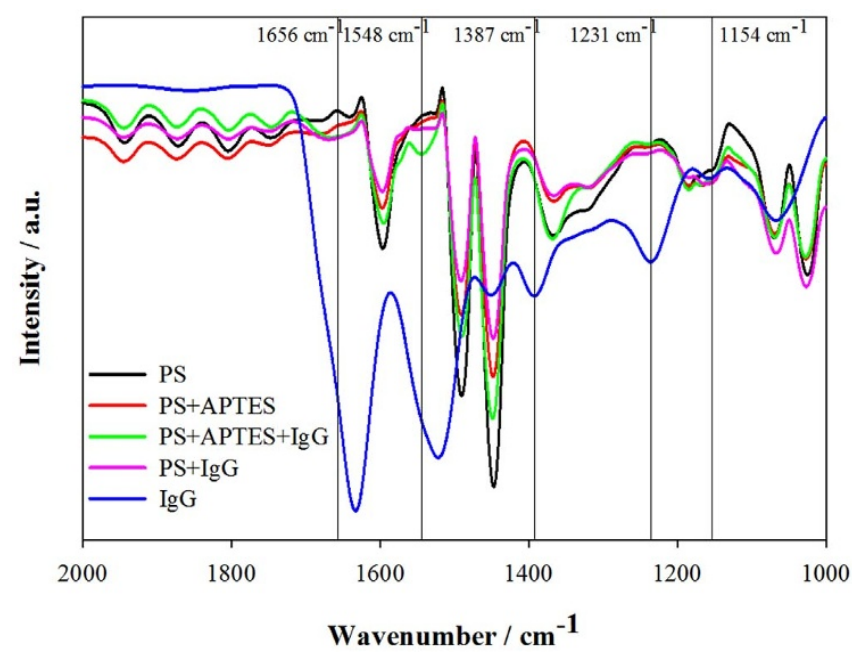

Figure 3 FTIR spectra pertaining to the APTES-functionalization and the immobilization of antibody on polystyrene (PS) surface.

The amount of APTES bound to PS is low, but consistent with the fact that PS lacks the polar, hydrogen bond accepting groups that promote the initial adsorption chain reaction postulated for the binding of APTES to different polymers with available surface hydroxyl groups ${ }^{24}$.

IgG may physically adsorb to the PS surface by interaction with the hydrophobic groups of antibody molecules without the need for APTES $^{25}$. When PS was immersed in a PBS solution of IgG, IgG bands were present in the purified PS+IgG FTIR spectrum (Figure 3, purple band). Since no coupling agents were employed to initiate the amide coupling between APTES and IgG, the binding of IgG to PS must be one of physical adsorption. The presence of water has two effects in the binding scheme of PS with APTES and IgG. Water can render the amine groups of APTES to be positively charged, allowing the APTES to bind to the anionic carboxylate groups of IgG through electrostatic interaction ${ }^{26}$. Water can also catalyze the polymerization of APTES as it hydrolyzes the ethoxy groups to give reactive silanol groups ${ }^{23}$, which would lead to the formation of Si-O-Si bonds detected in the PS+APTES+IgG FTIR spectrum.

PS was hydroxylated by $\mathrm{KOH}$ and followed by EDC coupling of IgGs as a covalent immobilization control in NCIF. The reactivity of PS with $\mathrm{KOH}$ is negligible as evident by very little change in the FTIR spectrum (Supplementary Figure S3). The amide bond formation between EDC-activated IgG and APTES displays two new bands at
$1552 \mathrm{~cm}^{-1}$ and $1642 \mathrm{~cm}^{-1}$ (Supplementary Figure S3, inset), which are mutually exclusive from the amide bands associated with the bound IgG. The peak at $1154 \mathrm{~cm}^{-1}$ reflects the polymerization of APTES (Si-O-Si bond) as described previously. Using anti-HFA Ab, the NCIF control offered no better analytical performance compared to the NIF (Supplementary Figure S4). It was reasoned that a lower $\mathrm{Ab}$ immobilization density was due to a decrease in hydrophobic character for the covalently coupled APTES-Ab entity.

The NIF is multisubstrate-compatible and adaptable to various commercial substrates including the modified MTP format (Supplementary Figure S5) ${ }^{27}$. The detection of HFA spiked in diluted human whole blood and plasma (Figure 2c) using the NIF exhibited similar precision as the commercial kit, showing its applicability for the determination of HFA in clinical and bioanalytical settings (Table 2). The NIF-based sandwich ELISA is the most sensitive immunoassay format in comparison to the commercial and previously developed sandwich ELISA formats for HFA (Table 3). The stability of Ab immobilized on MTPs was assessed over a two months period at $4^{\circ} \mathrm{C}$ in $0.1 \mathrm{M}$ PBS ( $\mathrm{pH}$ 7.4). No significant decrease in the $\mathrm{Ab}$ functional activity or FTIR signature was observed after eight weeks (Figure 2d, Supplementary Figure S6), attesting the leachproof Ab immobilization. Lastly, the NIF was applicable for detecting C-reactive protein (CRP), human albumin and human lipocalin-2 (Figure 4) with superior performance over the commercial kit.

\section{Discussion}

The NIF was developed through physical adsorption of APTES-polymer/Ab complexes onto a MTP in just $30 \mathrm{~min}$. The improved analytical performance, high simplicity and cost-effectiveness of the NIF was compared to commercial sandwich ELISA kits with high protein immobilization density, prolonged stability, high reproducibility and less biofouling and interferences ${ }^{28-34}$. The NIF, where APTES serves as a diluting and binding agent for anti-HFA capture $\mathrm{Ab}$ rather than $\mathrm{a}$ surface functionalization agent, demonstrates superior analytical performance and high stability.

With the NIF, only $0.5 \%$ APTES was used in the new procedure as compared to $2 \%$ APTES for the step-wise binding of Ab onto an APTES-functionalized PS surface ${ }^{17}$ in CovIF. The presence of the aqueous PBS buffer has two effects in the binding scheme of PS with APTES and Ab. Water can catalyze the polymerization of APTES as it hydrolyzes the ethoxy groups to reactive silanol groups (see Figure 1$)^{23}$. Confirmation of polymerization of APTES (Si-O-Si bond) is given by the band at $1154 \mathrm{~cm}^{-1}$ in the FTIR spectra of APTES-possessing species. There is the potential for polymerization of the APTES to form single and multilayers of APTES. In horizontal polymerization with respect to the $-\left(\mathrm{CH}_{2}\right)_{2} \mathrm{NH}_{2}$ plane, the binding is strong owing to ionic interactions of the two carboxylate groups of

Table 2 | Determination of spiked HFA concentrations in diluted human whole blood and plasma by NIF-and CIF-based sandwich ELISAs. The experiments were performed in triplicate, while the results are presented as mean \pm S.D.

\begin{tabular}{|c|c|c|c|}
\hline Sample matrix & Added conc. (in $\mathrm{ng} / \mathrm{mL}$ ) & NIF-based sandwich ELISA & CIF-based sandwich ELISA \\
\hline \multirow[t]{6}{*}{ Diluted human whole blood } & 20 & $20.1 \pm 0.19$ & $20.2 \pm 0.18$ \\
\hline & 10 & $10.2 \pm 0.16$ & $10.3 \pm 0.24$ \\
\hline & 5 & $5.0 \pm 0.17$ & $5.1 \pm 0.09$ \\
\hline & 1.2 & $1.3 \pm 0.06$ & $1.4 \pm 0.05$ \\
\hline & 0.6 & $0.5 \pm 0.03$ & $0.7 \pm 0.03$ \\
\hline & 0.3 & $0.3 \pm 0.01$ & $0.4 \pm 0.02$ \\
\hline \multirow{5}{*}{ Diluted human plasma } & 5 & $4.9 \pm 0.19$ & $4.7 \pm 0.18$ \\
\hline & 2.5 & $2.5 \pm 0.11$ & $2.7 \pm 0.12$ \\
\hline & 1.2 & $1.2 \pm 0.08$ & $1.3 \pm 0.09$ \\
\hline & 0.6 & $0.6 \pm 0.04$ & $0.5 \pm 0.03$ \\
\hline & 0.3 & $0.3 \pm 0.02$ & $0.3 \pm 0.01$ \\
\hline
\end{tabular}




\begin{tabular}{|c|c|c|c|}
\hline Manufacturer/Immunoassay Procedures & Antibody binding & Sensitivity (ng mL ${ }^{-1}$ ) & References \\
\hline New ELISA format (NIF) & One-step & 0.01 & This work \\
\hline Conventional ELISA & Passively adsorbed & 0.5 & This work \\
\hline RnD systems & Passively adsorbed & 0.37 & http://www.rndsystems.com/pdf/DY1 184.pdf \\
\hline Alpco Diagnostics & Passively adsorbed & 5.00 & http://www.alpco.com/pdfs/43/43-NSEHU-EO 1.pdf \\
\hline Immunology Consultants Laboratory, Inc. & Passively adsorbed & 6.25 & http://www.life-sciences.com.br/pdf/icllab.pdf \\
\hline Genway Biotech, Inc. & Passively adsorbed & 6.25 & $\begin{array}{l}\text { http://www.genwaybio.com/images/gw_tds/elisa_kits/40- } \\
\text { 374-130036.pdf }\end{array}$ \\
\hline Assay Pro & Passively adsorbed & 6.25 & http://www.assaypro.com/datasheet/eg3501_1.pdf \\
\hline
\end{tabular}

the $\mathrm{Ab}$ (heavy chain and/or light chain) with two adjacent amino groups of the APTES polymer network. Water can also render the amine groups of APTES to be positively charged, thereby allowing the APTES to bind to the anionic carboxylate groups of $\mathrm{Ab}$ via electrostatic interaction (Figure 1$)^{26}$. In addition, the silanol group is also capable of displaying intra and inter ionic interactions with the amino groups of APTES and Ab (heavy chain and/or light chain). The silanol and amino groups of APTES also display extensive

(a)

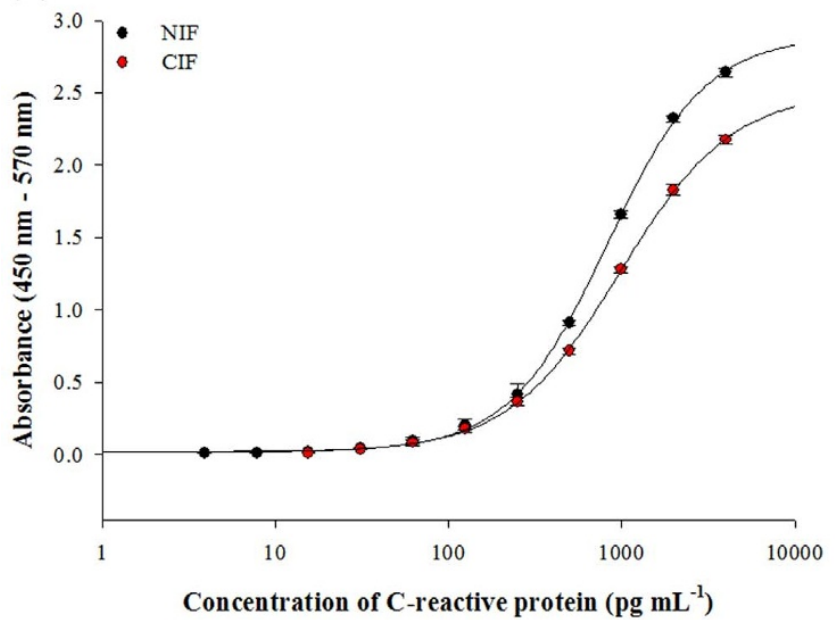

(c)

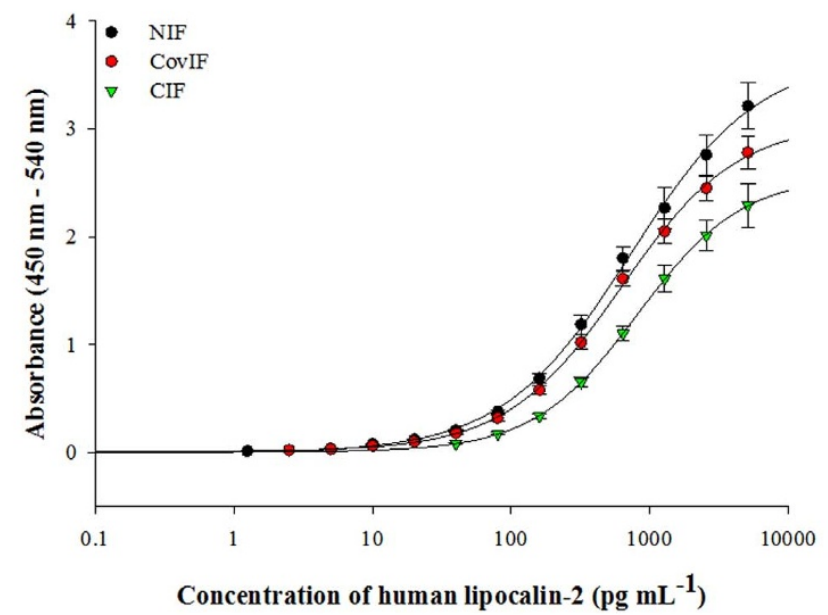

hydrogen bonding with the amino and carboxylic group of the $\mathrm{Ab}$ to form a stable APTES-Ab polymer network. One might anticipate a more favorable interaction between $\mathrm{Ab}$ and APTES in the liquid form compared to that of Ab with the APTES-functionalized surface. The orientation of bound $\mathrm{Ab}$ might also affect its bioanalytical performance.

Typically without $\mathrm{KOH} /$ plasma treatment, the number of APTES molecules bound to PS is low due to the lack of the polar, hydrogen

(b)

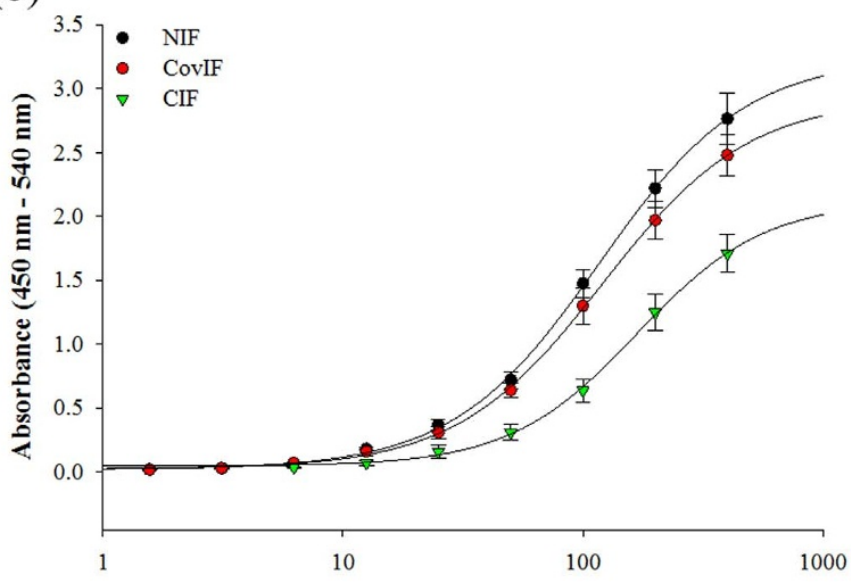

Concentration of human albumin $\mathrm{A}\left(\mathrm{ng} \mathrm{mL}^{-1}\right)$

Figure $4 \mid$ Immunoassays based the NIF for (a) C-reactive protein (CRP), (b) human albumin and (c) human lipocalin-2. The NIF-based immunoassays were compared with the CIF and CovIF-based sandwich ELISAs. All experiments were done in triplicate with the error bars representing the standard deviation. 
bond accepting groups that promote an initial adsorption chain reaction postulated for the binding of APTES to different polymers ${ }^{24}$. However, Ab may physically adsorb to the surface of PS by interaction with the hydrophobic groups of $\mathrm{Ab}$ molecules without the need for $\mathrm{APTES}^{25}$. Since the new procedure does not use crosslinking agents to initiate any amide coupling between APTES and Ab, physical adsorption could be attributed to the binding of $A b$ to the surface. Considering slight hydrophobicity of the aliphatic chain of APTES ${ }^{35}$, a higher Ab immobilization density could be achieved to promote greater physical adsorption of the pre-formed APTES polymer-Ab complex onto the PS surface. Based on the extreme simplicity and time saving granted by the NIF, the end-users can prepare the antiHFA capture Ab-bound plates just before their intended use. This obviates the need for storing Ab-prebound MTPs, which can lead to tremendous cost-savings and improved analytical performance due to the skipping of storage effects. The NIF is generic and applicable for detecting three selected biomarkers; therefore, this assay format will be of immense utility in the field of sandwich ELISA-based IVD kits.

\section{Methods}

Ab immobilization and HFA ELISA. The anti-HFA $(8 \mu \mathrm{g} / \mathrm{mL}$ in PBS $)$ was mixed with $1 \%$ APTES in the ratio of $1: 1(\mathrm{v} / \mathrm{v})$. Thereafter, the anti-HFA solution, with a final concentration of $4 \mu \mathrm{g} / \mathrm{mL}$ in $0.5 \%$ APTES, was added to the MTP wells and incubated for $30 \mathrm{~min}$ at room temperature. After washing with PBS, the anti-HFAbound MTP wells were blocked with $1 \%(\mathrm{v} / \mathrm{v})$ bovine serum albumin (BSA, diluted in $0.1 \mathrm{M}$ PBS, $\mathrm{pH}$ 7.4) for $30 \mathrm{~min}$ and washed with PBS. The anti-HFA-bound MTP wells were then incubated with various HFA concentrations $\left(4.9 \mathrm{pg} \mathrm{mL}^{-1}\right.$ to $20 \mathrm{ng}$ $\mathrm{mL}^{-1}$ ) for $1 \mathrm{~h}$ and washed with PBS. Thereafter, biotinylated anti-HFA (200 ng $\mathrm{mL}^{-1}$ ) was provided and incubated for $1 \mathrm{~h}$ followed by PBS washings. Subsequently, HRP-conjugated streptavidin, at a dilution of $1: 200$, was added to the MTP wells and incubated for $20 \mathrm{~min}$ followed by PBS washings. Such steps were performed at $37^{\circ} \mathrm{C}$. The 3,3',5,5' -tetramethylbenzidine (TMB) substrate was then added as per the manufacturer's guidelines, and the enzyme-substrate reaction was stopped after 20 min by adding $50 \mu \mathrm{L}$ of $2 \mathrm{~N} \mathrm{H}_{2} \mathrm{SO}_{4}$. The absorbance was measured at $450 \mathrm{~nm}$, taking $540 \mathrm{~nm}$ as the reference wavelength as per the manufacturer's guidelines. All experiments were carried out in triplicate and the absorbance of the blank $\left(0 \mathrm{ng} \mathrm{mL} \mathrm{m}^{-1}\right.$ HFA in 0.1 M PBS, pH 7.4) was subtracted from all assay values.

The conventional sandwich ELISA was performed as per the manufacturer's guidelines provided in the product information sheet without any modification. Various experimental process controls were employed to determine the efficiency of the BSA blocking, non-specific interactions of BSA with HFA, biotinylated anti-HFA and streptavidin-conjugated horseradish peroxidase (SA-HRP), and non-specific interaction of capture anti-HFA with biotinylated anti-HFA. The capture antibody being used in the commercial HFA ELISA kit is polyclonal mouse anti-HFA, while the detection antibody is monoclonal biotinylated goat anti-HFA. The commercial kit also states that the sandwich ELISA exhibits no cross-reactivity or interference with several recombinant human analytes (bone morphogenetic protein-2 (BMP-2), BMP-4, BMP-6, cathepsin V, matrix metalloproteinase-2 (MMP-2), MMP-9, transforming growth factor- $\beta 1$ (TGF- $\beta 1$ ), TGF- $\beta 2$ ) and recombinant mouse fetuin A.

All datasets were subjected to standard curve analysis using SigmaPlot software, version 11.2. The $\mathrm{EC}_{50}, R^{2}$ and Hill slope values were determined from the report generated by the software during standard curve analysis based on a four-parameter logistic function. The analytical sensitivity and LOD are calculated by the standard formulae, as mentioned below and further specified in the literature ${ }^{17,22,36,37}$.

$$
O D \cdot L O D=\text { Average } O D \cdot \text { Blank }-3\left(S D_{\cdot \text { Min.Analyte Conc. }}\right)
$$

$$
O D \cdot A S=\text { Average } O D \cdot \text { Blank }_{\text {B }}-3\left(S D_{\cdot \text { Blank }}\right)
$$

where $O D_{\cdot L O D}$ and $O D_{\cdot A S}$ are the optical densities corresponding to LOD and analytical sensitivity, respectively; $O D$. Blank is the optical density of the blank; and $S D_{\text {.Min }}$ Analyte Conc. and $S D$. Blank are the standard deviations of the minimum analyte concentration and the blank, respectively.

Buffers and solutions were prepared in Milli-Q deionized water. The dilution of all HFA assay components and BSA was made in $0.1 \mathrm{M}$ PBS, whereas KOH and APTES were diluted in deionized water. The HFA-spiked samples were prepared by admixing various concentrations of HFA in diluted human plasma and whole blood. The HFA dilution was made in BSA-preblocked glass vials, prepared by incubation with $1 \%(\mathrm{w} /$ v) BSA for $30 \mathrm{~min}$ to minimize analyte loss due to non-specific adsorption on sample tube surfaces and/or altered immunogenicity ${ }^{38}$. Deionized water and PBS washings were done five times with $300 \mu \mathrm{L}$ of the respective solutions, while $100 \mu \mathrm{L}$ was taken for other solutions, i.e. $1 \% \mathrm{KOH}$, anti-HFA solution (where anti-HFA was mixed with $1 \%$ APTES in the ratio of $1: 1(\mathrm{v} / \mathrm{v}))$, HFA, biotinylated anti-HFA, SA-HRP and TMB substrate. Unless otherwise indicated, the assay temperature and other protocols were maintained at $37^{\circ} \mathrm{C}$ using a thermostat while the absorbance was measured by a Tecan Infinite M200 Pro microplate reader. The details of the materials used and the characterization experiments performed are provided in the supplementary information.

1. Lim, P. et al. Usefulness of fetuin-A and C-reactive protein concentrations for prediction of outcome in acute coronary syndromes (from the French registry of acute ST-elevation non-ST-elevation myocardial infarction [FAST-MI]). Am. J. Cardiol. 111, 31-37 (2013).

2. Drake, R. R. et al. Lectin capture strategies combined with mass spectrometry for the discovery of serum glycoprotein biomarkers. Mol. Cell. Proteomics 5, 1957-1967 (2006).

3. Lim, P. et al. Fetuin-A is an independent predictor of death after ST-elevation myocardial infarction. Clin. Chem. 53, 1835-1840 (2007).

4. Sato, H. et al. Decreased levels of circulating $\alpha 2$-Heremans-Schmid glycoprotein/ fetuin-A (AHSG) in patients with rheumatoid arthritis. Intern. Med. 46, 1685-1692 (2007).

5. Weikert, C. et al. Plasma fetuin-A levels and the risk of myocardial infarction and ischemic stroke. Circulation 118, 2555-2562 (2008).

6. Honda, H. et al. Serum albumin, C-reactive protein, interleukin 6, and fetuin A as predictors of malnutrition, cardiovascular disease, and mortality in patients with ESRD. Am. J. Kidney. Dis. 47, 139-148 (2006).

7. Ketteler, M. et al. Association of low fetuin-A (AHSG) concentrations in serum with cardiovascular mortality in patients on dialysis: a cross-sectional study. Lancet 361, 827-833 (2003)

8. Jethwaney, D. et al. Fetuin-A, a hepatocyte-specific protein that binds Plasmodium berghei thrombospondin-related adhesive protein: a potential role in infectivity. Infect. Immun. 73, 5883-5891 (2005).

9. Srinivas, P. et al. Serum alpha 2-HS-glycoprotein is an inhibitor of the human insulin receptor at the tyrosine kinase level. Mol. Endocrinol. 7, 1445-1455 (1993).

10. Ix, J. H. et al. Association between human fetuin-A and the metabolic syndrome data from the heart and soul study. Circulation 113, 1760-1767 (2006).

11. Harris, V. K. et al. Cerebrospinal fluid fetuin-A is a biomarker of active multiple sclerosis. Mult. Scler. J. 19, 1462-1472 (2013).

12. Vashist, S. K., Dixit, C. K., MacCraith, B. D. \& O'Kennedy, R. Effect of antibody immobilization strategies on the analytical performance of a surface plasmon resonance-based immunoassay. Analyst 136, 4431-4436 (2011).

13. Kausaite-Minkstimiene, A., Ramanaviciene, A., Kirlyte, J. \& Ramanavicius, A. Comparative study of random and oriented antibody immobilization techniques on the binding capacity of immunosensor. Anal. Chem. 82, 6401-6408 (2010).

14. Vashist, S. K., Saraswat, M. \& Holthöfer, H. Development of a rapid sandwich enzyme linked immunoassay procedure for the highly sensitive detection of human lipocalin-2/NGAL. Procedia Chem. 6, 141-148 (2012).

15. Wong, L. S., Khan, F. \& Micklefield, J. Selective covalent protein immobilization: strategies and applications. Chem. Rev. 109, 4025-4053 (2009).

16. Jung, Y. et al. Photoactivable antibody binding protein: site-selective and covalent coupling of antibody. Anal. Chem. 81, 936-942 (2009).

17. Dixit, C. K., Vashist, S. K., MacCraith, B. D. \& O’Kennedy, R. Multisubstratecompatible ELISA procedures for rapid and high-sensitivity immunoassays. Nat. Protoc. 6, 439-445 (2011).

18. Lu, B., Smyth, M. R. \& O'Kennedy, R. Tutorial review. Oriented immobilization of antibodies and its applications in immunoassays and immunosensors. Analyst 121, 29R-32R (1996)

19. Shriver-Lake, L. C. et al. Antibody immobilization using heterobifunctional crosslinkers. Biosens. Bioelectron. 12, 1101-1106 (1997).

20. Vashist, S. K., Saraswat, M. \& Holthofer, H. Comparative study of the developed chemiluminescent, ELISA and SPR immunoassay formats for the highly sensitive detection of human albumin. Procedia Chem. 6, 184-193 (2012).

21. Vashist, S. et al. A multi-well plate for biological assays. WO 2010044083 A2, (2010).

22. Dixit, C. K. et al. Development of a high sensitivity rapid sandwich ELISA procedure and its comparison with the conventional approach. Anal. Chem. 82, 7049-7052 (2010).

23. Vandenberg, E. T. et al. Structure of 3-aminopropyl triethoxy silane on silicon oxide. J. Colloid Interface Sci. 147, 103-118 (1991).

24. Howarter, J. A. \& Youngblood, J. P. Surface modification of polymers with 3 aminopropyltriethoxysilane as a general pretreatment for controlled wettability. Macromolecules 40, 1128-1132 (2007).

25. Liu, X. et al. BSA-templated $\mathrm{MnO}_{2}$ nanoparticles as both peroxidase and oxidase mimics. Analyst 137, 4552-4558 (2012).

26. Wang, Z.-H. \& Jin, G. Silicon surface modification with a mixed silanes layer to immobilize proteins for biosensor with imaging ellipsometry. Colloids Surf. B 34, 173-177 (2004).

27. Vashist, S. K. Comparison of 1-ethyl-3-(3-dimethylaminopropyl)carbodiimide based strategies to crosslink antibodies on amine-functionalized platforms for immunodiagnostic applications. Diagnostics 2, 23-33 (2012).

28. Shah, R. et al. inventors; Medtronic Minimed, Inc., assignee. Long term analyte sensor array. US 7,577,470. 2009 Aug 18.

29. Shah, R., Reghabi, B., Gottlieb, R., Hoss, U. \& Mastrototaro, J. inventors; Medtronic Minimed, Inc, assignee. Analyte sensors and methods for making and using them. US20050272989 A1. 2005 Dec 8.

30. Shah, R. et al. inventors; Medtronic Minimed, Inc., assignee. Biosensors and methods for making and using them. US 7,813,780 B2. 2010 Oct 12 . 
31. Zheng, D. et al. Effect of 3-aminopropyltriethoxysilane on the electrocatalysis of carbon nanotubes for reagentless glucose biosensing. J. Nanopharmaceutics Drug Del. 1, 64-73 (2013).

32. Zheng, D., Vashist, S. K., Al-Rubeaan, K., Luong, J. H. T. \& Sheu, F. S. Mediatorless amperometric glucose biosensing using 3-aminopropyltriethoxysilanefunctionalized graphene. Talanta 99, 22-28 (2012).

33. Zheng, D., Vashist, S. K., Al-Rubeaan, K., Luong, J. H. T. \& Sheu, F. S. Rapid and simple preparation of a reagentless glucose electrochemical biosensor. Analyst 137, 3800-3805 (2012)

34. Zheng, D. et al. Graphene versus multi-walled carbon nanotubes for electrochemical glucose biosensing. Materials 6, 1011-1027 (2013).

35. Cunliffe, D., Smart, C., Alexander, C. \& Vulfson, E. Bacterial adhesion at synthetic surfaces. Appl. Environ. Microbiol. 65, 4995-5002 (1999).

36. Vashist, S. K. A sub-picogram sensitive rapid chemiluminescent immunoassay for the detection of human fetuin A. Biosens. Bioelectron. 40, 297-302 (2013).

37. Vashist, S. K. Graphene-based immunoassay for human lipocalin-2. Anal. Biochem. 446, 96-101 (2014).

38. Dixit, C. K., Vashist, S. K., MacCraith, B. D. \& O'Kennedy, R. Evaluation of apparent non-specific protein loss due to adsorption on sample tube surfaces and/ or altered immunogenicity. Analyst 136, 1406-1411 (2011).

\section{Author contributions}

S.K.V. proposed the developed sandwich ELISA procedure and one-step antibody immobilization strategy, and performed the immunoassay experiments. E.L. and S.H. conducted the characterization experiments, while E.M.S. and J.H.T.L. contributed in the design of experiments and research supervision. All the authors contributed to the drafting of this manuscript.

\section{Additional information}

Supplementary information accompanies this paper at http://www.nature.com/ scientificreports

Competing financial interests: The authors declare no competing financial interests. How to cite this article: Vashist, S.K., Schneider, E.M., Lam, E., Hrapovic, S. \& Luong, J.H.T One-step antibody immobilization-based rapid and highly-sensitive sandwich ELISA procedure for potential in vitro diagnostics. Sci. Rep. 4, 4407; DOI:10.1038/srep04407 (2014)

(i) $(-)$ This work is licensed under a Creative Commons AttributionNonCommercial-NoDerivs 3.0 Unported license. To view a copy of this license, visit http://creativecommons.org/licenses/by-nc-nd/3.0 